\title{
Einfluss von IT-Service-Management-Frameworks auf die IT-Organisation
}

\section{Eine empirische Studie zu Vorteilen, Herausforderungen und Prozessen}

Das Ziel dieses Beitrags besteht darin, ein besseres Verständnis für die Vorteile zu gewinnen, die durch eine Implementierung von ITIL entstehen. Die Studie vergleicht die Herausforderungen und Vorteile für Unternehmen auf verschiedenen Implementierungsebenen. Darüber hinaus wird auch die Anzahl der implementierten Prozesse auf den verschiedenen Ebenen betrachtet. Die Ergebnisse deuten darauf hin, dass die Wahrnehmung von Herausforderungen sinkt, sobald die Reife der Implementierung steigt. Die Ergebnisse zeigen darüber hinaus, dass durch eine Erhöhung der Implementierungsreife auch die Anzahl der realisierten Vorteile steigt. Der Beitrag gibt Einblicke in Bezug auf die Wahrnehmung der Effektivität von ITIL und präsentiert Implikationen für Praxis und Forschung.

DOI 10.1007/s11576-010-0257-8

\section{Die Autoren}

\author{
Mauricio Marrone $(\varangle)$ \\ C/O MGSM Research Office \\ Macquarie University \\ Building E14B \\ NSW 2109 Sydney \\ Australia \\ mmarron@uni-goettingen.de \\ Prof. Dr. Lutz M. Kolbe \\ Professur für \\ Informationsmanagement \\ Institut für Wirtschaftsinformatik \\ Platz der Göttinger Sieben 5 \\ 37073 Göttingen \\ Deutschland \\ Ikolbe@uni-goettingen.de
}

Eingegangen: 2010-05-01

Angenommen: 2010-11-05

Angenommen nach zwei Überarbeitungen durch Prof. Dr. Bichler.

Online publiziert: 2011-01-15
This article is also available in English via http://www.springerlink.com and http://www.bise-journal.org: Marrone M, Kolbe LM (2010) Impact of IT Service Management Frameworks on the IT Organization. An Empirical Study on Benefits, Challenges, and Processes. Bus Inf Syst Eng. doi: 10.1007/s12599-010-0141-5.

(C) The Author(s) 2011. Dieser Artikel ist auf Springerlink.com mit Open Access verfügbar

\section{Einleitung}

Im Zuge der Auflistung kritischer Erfolgsfaktoren für Informationssysteme (IS) im Jahr 1980 argumentiert Rockart (1982): „the first, and most obvious, IS critical success factor is service". Die ITAbteilungen in vielen Unternehmen konzentrierten sich zuvor auf die Produktion von Softwareanwendungen, während sich hingegen in den späten 1980erJahren ein Wandel zu einer serviceorientierten Arbeitsweise vollzog. In Bezug auf IT-Service-Management (ITSM) liegt der Schwerpunkt nicht auf der Entwicklung von IT-Anwendungen, sondern auf der Verwaltung von IT-Services.

In der Literatur existieren mehrere Studien zur Einführung von ITSM-
Frameworks sowie zu bestimmten serviceorientierten IT-Frameworks. Winniford et al. (2009) behaupten, dass etwa $45 \%$ aller US-Unternehmen ein ITSMFramework nutzen und $15 \%$ deren Nutzung planen. Das IT Governance Institute (2008) schätzt, dass die IT Infrastructure Library (ITIL) mit $24 \%$ das ITFramework mit der höchsten Akzeptanzrate ist, gefolgt von Control Objectives for Information und related Technology (CobiT) mit einer Akzeptanz von $14 \%$.

Zusätzlich zu den steigenden Akzeptanzraten von ITSM-Frameworks ist es notwendig, die durch IT-Services verursachten Kosten $\mathrm{zu}$ betrachten. ITDienstleistungen machen schätzungsweise $70 \%$ bis $80 \%$ der Ausgaben einer IT-Organisation aus (Orlov 2005). In der Praxis besteht ein Interesse daran, die möglichen Vorteile zu verstehen, die ein Unternehmen durch die Realisierung eines ITSM-Frameworks erreichen kann.

Die vorliegende empirische Studie konzentriert sich auf ITIL als das bekannteste ITSM-Framework. Der Forschungsbeitrag fokussiert auf das Verständnis für die Entstehung dieser Vorteile, wenn Unternehmen verstärkt durchgängig die Prozesse des ITIL-Modells umsetzen. Darüber hinaus ist die Wahrnehmung der Herausforderungen einer Implementierung von ITIL von Interesse sowie, wie bereits erwähnt, die Entwicklung der 
Wahrnehmung der Herausforderungen durch die Einführung des Modells im Unternehmen. Schließlich ist es notwendig, zu verstehen, wie die Umsetzung von ITIL-Prozessen die Reife der ITILImplementierung beeinflusst.

Bislang existieren keine wissenschaftlichen Untersuchungen $\mathrm{zu}$ diesem Thema und insbesondere eine groß angelegte internationale Studie wurde bisher nicht durchgeführt. Daher befasst sich der vorliegende Forschungsbeitrag damit, mithilfe empirischer Daten aus einer Umfrage mit führenden Unternehmen aus verschiedenen Branchen ein Verständnis für die folgenden Fragestellungen zu schaffen:

- Welche Auswirkungen hat die Gesamtzahl der implementierten Prozesse auf die Reife der ITIL-Implementierung?

- Wie werden die Herausforderungen auf verschiedenen Ebenen der Reife der ITIL-Implementierung wahrgenommen?

- Wie entwickelt sich die Gesamtzahl der realisierten Vorteile, wenn sich die Reife der ITIL-Implementierung erhöht?

Der vorliegende Beitrag beginnt mit einer Literaturstudie $\mathrm{zu}$ IT-ServiceManagement, ITIL-Prozessen sowie den Vorteilen und Herausforderungen der Implementierung. Anschließend folgt eine Beschreibung der verwendeten Forschungsmethodik. Die in unserer Umfrage gesammelten Daten werden mithilfe der Kruskal-Wallis- und des MannWhitney-Tests analysiert, um Vergleiche innerhalb der verschiedenen Implementierungsebenen vorzunehmen. Die Ergebnisse der Umfrage werden dann analysiert und diskutiert. Bevor die Limitationen der Studie und zukünftige Forschungsbereiche betrachtet werden, werden abschließend Schlussfolgerungen gezogen.

\section{Literaturreview}

ITSM stellt einen Teilbereich innerhalb der Service Sciences dar, die sich mit dem IT-Betrieb (IT-Operations) befassen (Galup et al. 2009). ITSM kann definiert werden als ,a set of processes that cooperate to ensure the quality of live IT services, according to the levels of service agreed to by the customer" (Young 2004). Conger et al. (2008) fügen hinzu: ITSM „focuses on defining, managing, and delivering IT services to support business goals and customer needs, usually in IT Operations“.
Serviceorientiertes IT-Management kann als eine Philosophie für eine Orientierung an Märkten, Services, Lebenszyklen und Prozessen verstanden werden (Zarnekow et al. 2005). Zunächst existiert eine Marktorientierung, die eine Kunden-Lieferanten-Beziehung anstelle einer Beziehung als Projektpartner impliziert. Zweitens besteht eine Serviceorientierung, was bedeutet, dass die Serviceprovider über Serviceportfolios anstelle von Projektportfolios verfügen. Diese Serviceportfolios beinhalten alle der durch den Provider angebotenen ITServices. Drittens liegt der Schwerpunkt auf dem Service-Lebenszyklus, wodurch ITSM einen methodischen Ansatz für die Verwaltung von IT-Services bietet - von der Gestaltung, der Implementierung, dem Betrieb bis hin zur kontinuierlichen Verbesserung. Dabei stehen nicht nur die technischen Aspekte der IT im Vordergrund, sondern es wird auch die Anpassung der durch die IT realisierten Dienste und Funktionen innerhalb der Organisation ermöglicht. Das Hauptaugenmerk des Managements von IT-Services liegt auf den Kosten des gesamten Lebenszyklus, nicht nur auf den Kosten der Entwicklung. Viertens besteht eine Prozessorientierung, damit die IT-Organisation an Prozessen und nicht an funktionalen Strukturen ausgerichtet ist.

Es existieren verschiedene Konzepte von ITSM-Frameworks. Die häufigste Methode ist ITIL, welches einen de-facto Standard für IT-Serviceprovider darstellt (Hochstein et al. 2005; IT Governance Institute 2008). Eine Vielzahl von ITSMFrameworks wurde auf Basis von ITIL als Referenz entwickelt, wie bspw. von Hewlett-Packard (HP ITSM Referenzmodell), IBM (IT Process Model) und das MOF von Microsoft (van Bon et al. 2007).

Die aktuelle Version von ITIL, Version 3, wurde im Mai 2007 veröffentlicht. Sie besteht aus 26 Abschnitten, die in den folgenden fünf Phasen des Lebenszyklus enthalten sind: Service Strategy, Service Design, Service Transition, Service Operation und Continual Service Improvement. Die frühere Version, Version 2, verfügt insgesamt über zehn Prozesse in zwei wesentlichen Bereichen: Service Support und Service Delivery. Es existieren darüber hinaus noch weitere Bereiche mit geschäftlichen Leitlinien, wobei sich dieser Beitrag jedoch auf diese zwei wichtige Bereiche konzentriert.

Die wissenschaftliche Forschung $\mathrm{zu}$ ITSM befindet sich in einer frühen Pha- se trotz zahlreicher Publikationen, vor allem in Praktikerzeitschriften. Bestehende wissenschaftliche Literatur präsentiert die Beschreibung der Bereiche, die im Rahmen von ITIL dokumentiert sind (Cervone 2008; Hendriks und Carr 2002) oder analysiert Anwender von ITIL anhand von Fallstudien (Hochstein et al. 2005; Cater-Steel et al. 2006b; Marrone et al. 2010). Wenige Forscher haben sich bisher mit dem Thema der Vorteile, Herausforderungen der Umsetzung und der Effektivität von ITIL auseinandergesetzt. Tabelle 1 stellt die relevanten wissenschaftlichen Forschungsbeiträge zusammen.

Eine Zusammenfassung der Vorteile von ITSM-Frameworks in der Literatur wird in Tab. 2 dargestellt. Tabelle 3 bietet eine Zusammenfassung der Herausforderungen bei der Umsetzung ITSMFrameworks.

Von zahlreichen Forschern, darunter Cater-Steel et al. (2007) und Marrone et al. (2010), wurde das Reifegradmodell verwendet. Die Ebenen des Reifegradmodells basieren in diesen Studien auf dem Modell von CobiT und Capability Maturity Model Integration (CMMI). Diese Ebenen sind als Profile von IT-Prozessen vorgesehen und werden von Unternehmen als Beschreibung ihres aktuellen $\mathrm{Zu}$ stands identifiziert.

Bis heute existieren keine Untersuchungen, die die Vorteile, Herausforderungen und die Umsetzung von Prozessen sowie deren Zusammenhang mit der Reife der ITIL-Implementierung ermitteln. Abgesehen davon wurde das Instrument einer groß angelegten Umfrage für verschiedene Länder bisher nicht eingesetzt.

\section{Forschungsdesign}

Dieser Abschnitt beschreibt die einzelnen Einführungsniveaus, auch als Reifegradmodell bezeichnet, sowie die in diesem Beitrag untersuchten Thesen. Für die drei in der Einleitung aufgeführten Fragen werden insgesamt fünf Thesen beschrieben. Diese werden im Folgenden dargestellt.

\subsection{Ebenen des Reifegradmodells}

Das Reifegradmodell ist unterteilt in verschiedene Stufen, die von nicht existent (0) bis hin $\mathrm{zu}$ optimiert (5) reichen. 
Tab. 1 Relevante Forschung zu ITSM und ITIL

\begin{tabular}{|c|c|c|}
\hline Autor und Jahr & Ansatz & In der Studie angesprochene Aspekte \\
\hline Hochstein et al. (2005) & Qualitativ & $\begin{array}{l}\text { Nennt vier Vorteile: Verbesserung der Qualität von IT-Services, Effizienz und } \\
\text { Optimierung von Prozessen und Transparenz, Vergleichbarkeit durch } \\
\text { Prozessdokumentation und Prozessüberwachung. Nennt sechs Erfolgsfaktoren } \\
\text { bei der Umsetzung von ITSM-Frameworks: Demonstration von „quick wins“, } \\
\text { Streben nach ständiger Verbesserung, Marktkampagnen zur Schaffung von } \\
\text { Akzeptanz und Verständnis, Unterstützung des Managements, Schulung und } \\
\text { Bildung von virtuellen Teams, sodass „neue“ Prozesse gleichzeitig mit den } \\
\text { operativen Tätigkeiten entwickelt werden. }\end{array}$ \\
\hline
\end{tabular}

Potgieter et al. (2005) Qualitativ

Brenner (2006)

Cater-Steel et al.

(2006a)

Cater-Steel et al.

(2006b)

Spremic et al. (2008)

Cervone (2008)

Marrone et al. (2010)

Tan et al. (2009)

Pollard und Cater-Steel (2009a, 2009b)

Galup et al. (2009)

Iden und Langeland

(2010)
Qualitativ

Qualitativ

Konzeptionell und Quantitativ

Qualitativ

Konzeptionell

Qualitativ

Qualitativ

Konzeptionell

Qualitativ
Untersucht die Wirkung der Implementierung von ITIL auf die

Kundenzufriedenheit und Servicequalität. Die Forscher vermuten, dass am Ort der Untersuchung, einer großen Serviceeinrichtung für IKT in Südafrika, eine direkte Korrelation zwischen Kundenzufriedenheit, Servicequalität und Nutzung von ITIL vorliegt.

Schlägt Möglichkeiten vor, wie der ITIL-Prozess effizient mit prozessorientierten Werkzeugen realisiert werden kann, bspw. mithilfe von Workflow-Management-Systemen.

Stellt Prozesse wie ITIL, CobiT, CMMI und ISO 9001 dar und beschreibt mögliche Motivationen und Herausforderungen für ihre Einführung.

Beschreibt die Herausforderungen der Einführung von ITIL mithilfe der folgenden vier Faktoren: Mangel an Managementunterstützung, kultureller Wandel in Bezug auf Widerstand, Verzögerungen bei der Auswahl eines geeigneten Werkzeugs und Managementprobleme in Bezug auf Ressourcen, wie bspw. Zeit, Personal und Geld.

Überwacht einen IT-Serviceprovider in Kroatien und wendet verschiedene Key Performance Indikatoren (KPI) vor und nach der Implementierung einer Reihe von ITIL-Prozessen an. Die Studie ergibt, dass der IT-Serviceprovider Verbesserungen erreichen konnte, die der Implementierung von ITIL zuzurechnen sind.

Bietet einen Überblick über ITIL und schlägt die folgenden drei Vorteile vor: Kostensenkung, Verbesserung der Kundenzufriedenheit und Steigerung der Produktivität der IT-Abteilung.

Identifiziert sechs Faktoren, die als Vorteile einer ITIL-Einführung verstanden werden: Verbesserung der Kundenzufriedenheit, Verbesserung der internen Abläufe, Standardisierung von Prozessen, Verbesserung der Servicequalität, Steigerung der Effizienz und Verbesserung des Return on Investment.

Gibt Aufschluss über die Herausforderungen der Implementierung. Konzentriert sich auf KEF und kommt zu dem Schluss, dass ein geeigneter Führungsstab, eine angemessene Change-Management-Strategie, eine enge Beziehung mit mehreren Anbietern und eine effektive Projektsteuerung entscheidende Faktoren für die Umsetzung sind.

Folgende KEF werden identifiziert: Unterstützung der Unternehmensführung, abteilungsübergreifende Kommunikation und Kollaboration, Einsatz von Beratern, Ausbildung und sorgfältige Auswahl von Software, die Schaffung einer ITIL-freundlichen Kultur, Prozesse als Priorität und kundenorientierte Metriken.

Gibt einen Überblick über ITSM, dessen globale Auswirkungen und aktuelle Initiativen.

Untersucht die wichtigsten Faktoren für eine erfolgreiche Einführung von ITIL. Die wichtigsten Faktoren sind Managementunterstützung, Kompetenz und Ausbildung, Information und Kommunikation, Stakeholder-Engagement und die Fähigkeit, die Unternehmenskultur zu ändern.
Sie wurden ursprünglich vom CMMFramework und später von CobiT verwendet. Die vorliegende Studie verwendet die gleichen Reifegrade, die im Rahmen dieser Frameworks vorgeschlagen werden. Auf der untersten Ebene des
Reifegradmodells werden die Managementprozesse überhaupt nicht angewendet. Auf der folgenden Ebene, als anfänglich bezeichnet, sind Prozesse adhoc und desorganisiert. Die zweite Stu$\mathrm{fe}$, innerhalb derer die Prozesse intui- tiv sind und in den meisten Fällen deterministische und wiederholbare Ergebnisse erzielen, wird als wiederholbar bezeichnet. Auf Ebene drei sind Prozesse dokumentiert und bieten Standardverfahren sowie klar definierte Ergebnis- 
Tab. 2 Zusammenfassung der Vorteile der Implementierung von ITSM-Frameworks

\begin{tabular}{lllll}
\hline Verbesserung von... & $\begin{array}{l}\text { Hochstein et al. } \\
(2005)\end{array}$ & Potgieter et al. (2005) & Marrone et al. (2010) & $\begin{array}{l}\text { Cater-Steel et al. } \\
(2007,2008)\end{array}$ \\
\hline $\begin{array}{l}\text { Servicequalität } \\
\text { Standardisierung von }\end{array}$ & $\times$ & $\times$ & $\times$ & $\times$ \\
Services & $\times$ & $\times$ & $\times$ \\
Kundenzufriedenheit & & $\times$ & $\times$ \\
Return on Investment & & & $\times$ \\
Business-IT-Alignment & & & $\times$ \\
Reduzierung der & & & $\times$ \\
IT-Ausfallzeiten & & & $\times$ \\
Abläufen durch die & & & $\times$ \\
Implementierung einer & & & $\times$ \\
Best Practice & & & $\times$ \\
Kontrolle finanzieller & & & $\times$ \\
Beiträge & & & $\times$ \\
Call-Fix-Rate & & & $\times$ \\
Moral des IT-Personals & & & $\times$ \\
\hline
\end{tabular}

Tab. 3 Herausforderungen der Implementierung eines ITSM-Frameworks

\begin{tabular}{|c|c|c|c|c|c|}
\hline & $\begin{array}{l}\text { Hochstein et al. } \\
(2005)\end{array}$ & Tan et al. (2007) & $\begin{array}{l}\text { Cater-Steel et al. } \\
(2007,2008)\end{array}$ & $\begin{array}{l}\text { Iden und } \\
\text { Langeland } \\
(2010)\end{array}$ & $\begin{array}{l}\text { Pollard and } \\
\text { Cater-Steel } \\
(2009 a, 2009 b)\end{array}$ \\
\hline $\begin{array}{l}\text { Mangel an Unterstützung durch die } \\
\text { Geschäftsleitung }\end{array}$ & $\times$ & $\times$ & $\times$ & $\times$ & $\times$ \\
\hline $\begin{array}{l}\text { Verständnis der Ziele von ITIL im } \\
\text { Geschäftsbereich }\end{array}$ & & & $\times$ & $\times$ & \\
\hline $\begin{array}{l}\text { Mangel an Ressourcen (Zeit oder } \\
\text { Personen) }\end{array}$ & & & & & $\times$ \\
\hline $\begin{array}{l}\text { Mangelnde interne } \\
\text { Fähigkeiten/Kenntnisse in Bezug auf } \\
\text { ITIL }\end{array}$ & $\times$ & $\times$ & $\times$ & $\times$ & $\times$ \\
\hline $\begin{array}{l}\text { Fehlende Finanzierung/Kosten der } \\
\text { Einführung }\end{array}$ & $\times$ & & $\times$ & & \\
\hline $\begin{array}{l}\text { Organisatorischer/Kultureller } \\
\text { Widerstand gegen Veränderungen }\end{array}$ & $\times$ & & & $\times$ & $\times$ \\
\hline $\begin{array}{l}\text { Erhalt der dynamischen } \\
\text { Entwicklung/Fortschritt stagniert }\end{array}$ & & (Planvorgaben) & $\times$ & & $\times$ \\
\hline
\end{tabular}

se/Leistungen. Diese Stufe wird als definiert bezeichnet. Die vierte Stufe (kontrolliert), basiert auf kritischen Erfolgsfaktoren, wobei Key Performance Indikatoren (KPIs) zur Quantifizierung der Prozessleistung definiert und kontinuierlich gemessen werden. Der höchste Reifegrad wird als optimiert bezeichnet. Hier wurde der Zyklus der kontinuierlichen Verbesserung umgesetzt und basiert auf Kennzahlen und internen Audits. Auf dieser Ebene ist der Prozess in Bezug auf Effektivität, Effizienz und Compliance verbessert. Unternehmen würden diese Stufen im Rahmen der Beschreibung ihres aktuellen Zustands identifizieren.

\subsection{Implementierte Prozesse und Reifegrad}

Das Verhältnis zwischen der Anzahl der implementierten Prozesse und der Reifegrade ist von Interesse für den vorliegenden Forschungsbeitrag. Um die Wirkung der Gesamtzahl der durchgeführten Prozesse auf den Reifegrad der Implementierung zu verstehen, wurden zunächst zwei mögliche Thesen aufgestellt. Jedoch wurde nur eine von diesen von den Autoren ausgewählt. Die erste mögliche These ist, dass Unternehmen Prozesse auswählen und implementieren, die ihrer Meinung nach die größten Vortei- le für ihr Unternehmen bieten oder Prozesse, die ihnen dabei helfen, konkrete Defizite zu beheben. Gammelgård et al. (2007) legen nahe, dass Unternehmen dazu neigen, diejenigen Prozesse einzuführen, die sie am meisten benötigen. Tuttle und Vandervelde (2007) argumentieren, dass während der Umsetzung von GeschäftsprozessmanagementFrameworks nicht alle Prozesse berücksichtigt werden. Daher würden die Unternehmen, die nur einige der Prozesse implementiert haben, den Reifegrad ihrer ITIL-Implementierung auf Basis der Reife dieser implementierten Prozesse ange- 
ben, anstelle der Reife des gesamten ITILFrameworks.

Umgekehrt besteht eine weitere mögliche These darin, dass die Reife der ITILImplementierung auf dem gesamten Lebenszyklusmodell beruht. Deshalb basiert die Wahrnehmung des Reifegrades eher auf dem vollständigen ITIL-Modell, anstelle der Reife der implementierten Prozesse. Daraus folgt, dass je mehr ITILProzesse von den Unternehmen implementiert werden, desto höher auch die Reife der ITIL-Implementierung in diesen Unternehmen ist. Im vorliegenden Forschungsprojekt wird die letztere These unterstützt. Da die Anzahl der Prozesse je nach Version variiert, wird jede Version separat analysiert. Die These lautet:

T1: Es besteht ein positiver Zusammen-

hang zwischen den implementierten

Prozessen und der wahrgenommen Reife der ITIL-Implementierung.

\subsection{Wahrgenommene Herausforderungen und Reifegrad}

Diese Forschung zielt darauf ab, zu verstehen, welche Auswirkungen vorhandene Reifegrade auf die wahrgenommenen Herausforderungen der Implementierung haben. Die These der Wirkung der wahrgenommenen Herausforderungen auf den Reifegrad basiert auf dem Modell des Lernkurveneffekts. Die Lernkurve, auch als Erfahrungskurve bezeichnet, stellt ein Phänomen dar, das ursprünglich von Wright (1936) beobachtet wurde. Wright argumentiert, dass Lernen für die Produktion von jeglichen Waren oder Dienstleistungen auftreten kann. Durch Anwendung dieses Modells auf unsere These können wir davon ausgehen, dass die Organisation Erfahrungen zu dem Umgang mit Herausforderungen erlangt und effizienter wird, da sie in ihrem Lernen fortschreitet, sodass die Wahrnehmung der Herausforderungen im Lauf der Zeit nachlässt. Dementsprechend lautet unsere zweite These wie folgt:

T2: Es besteht eine negative Beziehung zwischen Reifegraden der ITILImplementierung und der wahrgenommenen Herausforderungen der Implementierung.

\subsection{Anzahl der realisierten Vorteile und Reifegrade}

Die nächste Frage zielt auf das Verständnis der Gesamtzahl der realisierten Vorteile durch die Implementierung von
ITIL für jedes Unternehmen ab. Anstatt sich auf die einzelnen Vorteile zu konzentrieren, nach denen gefragt wurde, liegt der Fokus dieser Forschung darin, die Gesamtzahl der realisierten Vorteile für die Unternehmen zu untersuchen. Folglich werden für jedes Unternehmen die realisierten Vorteile aufsummiert. Der gleiche Ansatz wird für die Anzahl der Vorteile verwendet, die durch Metriken unterstützt werden sowie für die Anzahl der Vorteile, die von den Geschäftsbereichen wahrgenommen wurden.

Im vorliegenden Beitrag wird die Ansicht vertreten, dass die Vorteile von ITIL zunächst durch die IT festgestellt werden und dass Metriken zum Beleg der Vorteile in früheren Stadien nicht zur Verfügung stehen. $\mathrm{Zu}$ späteren Reifegraden werden diese Vorteile von Metriken unterstützt und werden dann auch von den Geschäftsbereichen wahrgenommen.

Für diese These wird das Gesetz des abnehmenden Ertrags in die Betrachtung mit einbezogen. Dieses schlägt vor, dass die Bemühungen im Zuge einer kontinuierlichen Verbesserung für ein bestimmtes Projekt oder Ziel zu einem Rückgang der Effektivität führen, sobald ein bestimmter Grad der Ergebniserreichung erlangt ist (Drucker et al. 1998). Mit anderen Worten: Nach einem gewissen Maß an Standardisierung führt die Erhöhung der Standardisierung zu weiteren Vorteilen. Folglich wird die folgende These vorgeschlagen:

T3a: Es besteht ein positiver Zusammenhang zwischen Reifegraden der ITILImplementierung und der wahrgenommen realisierten Vorteile.

Wir erwarten auch, dass die Zahl der realisierten Vorteile, die durch Metriken unterstützt werden, erst zu späteren Reifegraden und nicht zu den früheren Reifegraden sichtbar wird. Dem Verständnis der Autoren zufolge wird im Falle von späteren Reifegraden von Unternehmen erwartet, Metriken regelmäßig zu nutzen. Insbesondere besteht das Interesse an dieser These nicht darin, zu sehen, ob Unternehmen Metriken per se nutzen, sondern vielmehr, ob die verwendeten Metriken die Wahrnehmung der erreichten Vorteile unterstützen können. Mit anderen Worten: Es ist wichtig, zu verstehen, ob IT-Führungskräfte die Vorteile der ITIL-Implementierung mit dem Einsatz von Metriken quantifizieren können. In ähnlicher Weise kann das Unternehmen die Vorteile der ITILImplementierung auf den späteren Ebenen der Akzeptanz erkennen, möglicher- weise aufgrund eines besseren BusinessIT-Alignments. Daher werden die folgenden Thesen vorgeschlagen:

T3b: Es besteht ein positiver Zusammenhang zwischen Reifegraden der ITILImplementierung und der Nutzung von Metriken, um die realisierten Vorteile zu messen.

T3c: Es besteht ein positiver Zusammenhang zwischen Reifegraden der ITILImplementierung und der Wahrnehmung der realisierten Vorteile durch den Geschäftsbereich.

\section{Methodik}

Der Fragebogen wurde online in den Monaten April und Mai 2009 zur Verfügung gestellt. Eine Einladung zur Umfrage wurde per E-Mail an Personen gesendet, die auf den Mailinglisten von Hornbill und des IT-Service-ManagementForums (itSMF) in den USA und Großbritannien stehen. Darüber hinaus wurde die Umfrage in einem Versuch, die Ergebnisse dieser Forschung zu erweitern, in verschiedenen Internetgruppen und foren angekündigt, die sich einzig mit dem Thema ITIL befassen. Die Zielteilnehmer der Umfrage sind Experten in ITIL in ihrer Organisation, die umfassend an der Implementierung von ITIL beteiligt sind.

Die Struktur des Fragebogens adressiert viele Aspekte von ITIL, dessen Einführung, Verwendung, Implementierung und Reife sowie die Effektivität von Prozessen und realisierten Vorteilen. Er umfasst zudem die Themen Business-ITAlignment und Service-Desk-Nutzung. Die Erhebung beinhaltete Fragen, für deren Antworten Likert-Skalen, nominale Skalen und offene Fragen verwendet wurden.

Zunächst wurden die Befragten gebeten, die Wahrnehmung der Reife ihres ITIL-Prozesses auf einer auf CobiT und CMMI basierenden Skala einzuordnen.

Die Befragten wurden auch gebeten, anzugeben, welche Version von ITIL sie implementiert haben. Darauf aufbauend wurden sie aufgefordert, die Prozesse auszuwählen, die sie unter Berücksichtigung ihrer ITIL-Version umgesetzt haben. Alle Prozesse wurden in der Umfrage aufgeführt.

Darüber hinaus wurden die Befragten nach ihrer Wahrnehmung der Herausforderungen der ITIL-Implementierung befragt. Sie wurden gebeten, Herausforderungen auf einer Skala von 1 bis 5 
Tab. 4 Profil der befragten Unternehmen $(n=491)$

\begin{tabular}{|c|c|c|c|c|c|}
\hline Branche & $\%$ & Länder & $\%$ & Zahl der Standorte & $\%$ \\
\hline Technologie & 31 & Großbritannien & 52 & $10+$ & 69 \\
\hline Öffentlichkeit & 23 & USA & 36 & $2-5$ & 18 \\
\hline Finanzen und Banken & 18 & Kanada & 1 & $6-10$ & 7 \\
\hline Freiberufler & 5 & Indien & 1 & 1 & 6 \\
\hline Fertigung & 5 & Irland & 1 & & \\
\hline Handel und Vertrieb & 5 & Sonstige & 9 & & \\
\hline Sonstige & 4 & & & & \\
\hline Energieversorgung & 3 & Zahl der Beschäftigten & $\%$ & Rolle des Beschäftigten & $\%$ \\
\hline Unterhaltung und Gastronomie & 2 & & & & \\
\hline Gesundheitswesen & 2 & $10000+$ & 40 & IT-Manager & 32 \\
\hline \multirow[t]{5}{*}{ Telekommunikation } & 2 & $1001-5000$ & 21 & Prozessspezifischer Manager & 22 \\
\hline & & $5001-10000$ & 16 & Service-Delivery-Manager & 17 \\
\hline & & $501-1000$ & 9 & IT-Direktor - Organisationsebene & 14 \\
\hline & & $101-500$ & 8 & Hilfe/Service-Desk-Manager & 11 \\
\hline & & $<100$ & 6 & Hilfe/Service-Desk-Mitarbeiter & 4 \\
\hline
\end{tabular}

zu bewerten, wobei 1 keine Herausforderung und 5 große Herausforderung bedeutete. Die untersuchten Herausforderungen, die auf Basis unserer Literaturstudie gesammelt und in Tab. $3 \mathrm{zu}$ sammengefasst wurden, sind der Mangel an Unterstützung durch die Geschäftsleitung, das geschäftliche Verständnis der Ziele von ITIL, der Mangel an Ressourcen (Zeit oder Personen), mangelnde interne Kenntnisse/Fähigkeiten in Bezug auf ITIL, fehlende Finanzierung/Kosten der Einführung, organisatorischer/kultureller Widerstand gegen Veränderungen sowie der Erhalt der dynamischen Entwicklung/Fortschritt stagniert.

Schließlich sollten die Befragten die realisierten Vorteile auswählen, die durch die Einführung von ITIL erreicht werden konnten. Die in der Umfrage aufgeführten Vorteile sind Verbesserungen: der Service-Qualität, der Kundenzufriedenheit, als Resultat standardisierter Prozesse, der Interaktion von IT mit dem Rest des Unternehmens, durch Reduzierung der IT-Ausfallzeiten, der Rendite für die IT-Ausgaben (ROI), durch Anwendung der Best-Practice-Erfahrungen anderer, bei der Messung des finanzielle Beitrags der IT für das Unternehmen, der Call-Fix-Rate und der Moral der ITMitarbeiter. Diese Vorteile sind in Tab. 2 dargestellt. Wenn die Befragten einen dieser Vorteile realisiert hatten, konnten sie wählen, ob diese Vorteile durch Metriken unterstützt wurden und ob das Un- ternehmen die Verbesserungen wahrgenommen hatte.

\section{Ergebnisse}

\subsection{Profil der Befragten}

Mehr als 5.000 Einladungen wurden an die Mitglieder des itSMF UK und US sowie an die Mailingliste von Hornbill geschickt. Von den 784 ITFührungskräften, die die Umfrage starteten haben 503 die Befragung abgeschlossen und abgeschickt. Zwölf Antworten wurden als ungültig identifiziert und aus der statistischen Analyse ausgeschlossen. Nur zum Teil ausgefüllte Fragebögen wurden nicht in der Studie verwendet. Vier Fünftel derjenigen, die die Umfrage abgeschlossen hatten, waren Teilnehmer, die direkt zur Umfrage eingeladen wurden, während die restlichen Teilnehmer durch den Eintrag in Foren gewonnen werden konnten. Die Rücklaufquote der Einladungen zur Umfrage betrug acht Prozent.

Die Teilnehmer wurden nach der Branche ihres Unternehmens, der Anzahl der durch die IT unterstützten Standorte, der Anzahl der Mitarbeiter im Unternehmen sowie deren Titel und deren Einsatzort befragt. Tabelle 4 stellt das Profil der Befragten dar. In der Stichprobe stammen rund drei Viertel der Befragten aus den Branchen Technologie, Öffentlichkeit und dem Finanz- und Bankensektor. Andere genannte Sektoren umfassen bspw. die Branchen Freiberufler, Handel und Fertigung.

Fast $70 \%$ der Befragten nannten zehn oder mehr Standorte, die durch eine zentrale IT unterstützt werden und knapp $20 \%$ gaben zwei bis fünf Standorte an. Fast $45 \%$ der Befragten arbeitet in einem Unternehmen mit mehr als 10.000 Mitarbeitern. Die Aufgabengebiete der Befragten werden ebenfalls in der Tab. 4 angegeben. Bei den Teilnehmern der Umfrage waren $33 \%$ IT-Manager, während es sich bei $23 \%$ um prozessspezifische Manager handelte. Durch den Einsatz von Mailinglisten und die Entsendung von Einladungen zur Umfrage auf englischsprachigen Websites, kam die Mehrheit der Antworten aus Großbritannien (52\%) und den USA (36\%), während verschiedene weitere Antworten aus Kanada, Indien und Irland stammen.

Abbildung 1 zeigt die Implementierungsreife von ITSM-Frameworks für alle Teilnehmer. Wie der Abb. 1 zuentnehmen ist, ordnet mehr als die Hälfte der Befragten ihre Implementierungen entweder auf Stufe 2 (wiederholbar) mit $32 \%$ oder auf Stufe 3 (definiert) mit $25 \%$ ein, während sich eine Minderheit an den Extremen einordnete, Stufe 1 (anfänglich) mit $13 \%$ und Stufe 5 (optimiert) mit $11 \%$.

\subsection{Empirischer Befund}

Für jede Variable wurde eine explorative Analyse durchgeführt, um die Normalität 


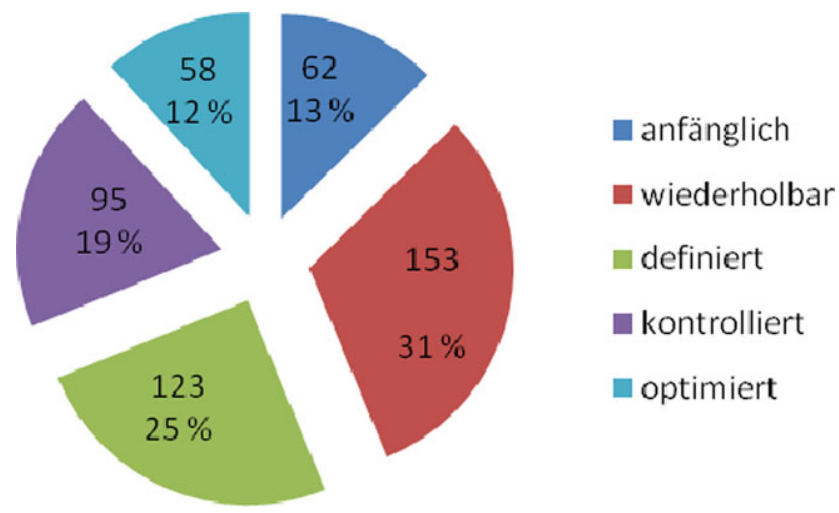

Abb. 1 Reife der ITIL-Implementierung in den befragten Unternehmen $(n=491)$

zu ermitteln. Sowohl der KolmogorovSmirnov- als auch der Shapiro-WilkTest wiesen Signifikanz für die Versionen von ITIL $(p<0,001)$, für alle Variablen, die die Herausforderungen der ITIL-Einführung beschreiben $(p<0,001)$ und für die durch ITIL realisierten Vorteile $(p<0,001)$ auf. Da die Daten nicht normalverteilt waren, wurde der Kruskal-Wallis-Test, eine nichtparametrische einseitige Varianzanalyse, genutzt, um die Daten zu analysieren. Wenn die Daten auf Basis des KruskalWallis-Tests signifikante Unterschiede zwischen den Gruppen zeigten, wurde der Mann-Whitney- $U$-Test angewandt.

$\mathrm{Da}$ es für die Studie von Relevanz ist, wie die genannten Variablen durch die Erhöhung der ITILImplementierung beeinflusst werden, wurden die Testmaßnahmen sorgsam ausgewählt. Der Mann-Whitney- $U$ Test erhöht die Typ-I-Fehlerrate, sodass die Wahl der getroffenen Vergleiche sorgfältig durchgeführt wurde.

Die vorgeschlagenen Vergleiche sind zwischen den ersten und mittleren Reifegraden der ITIL-Implementierung, den ersten und letzten Reifegraden der ITIL-Implementierung sowie zwischen den mittleren und letzten Reifegraden der ITIL-Implementierung. Daher wurden die folgenden drei Tests durchgeführt:

- Test 1: Reifegrad 1 (anfänglich) verglichen mit Reifegrad 3 (definiert)

- Test 2: Reifegrad 1 (anfänglich) verglichen mit Reifegrad 5 (optimiert)

- Test 3: Reifegrad 3 (definiert) verglichen mit Reifegrad 5 (optimiert)

Da drei Tests durchgeführt wurden, wurde eine Bonferroni-Korrektur angewandt. Durch diese Korrektur, anstelle der Verwendung des kritischen Signifikanzniveaus von 0,05, wiesen alle Effekte ein Signifikanzniveau von 0,0167 auf. Alle resultierenden p-Werte verwenden 2-Tailed Monte-Carlo p-Werte mit einem Konfidenzniveau von 99 $\%$ und einer Reihe von Samples mit 10.000. Dieses Verfahren wurde aufgrund der großen Stichprobe eingesetzt.

Zusätzlich wurde der JonckheereTerpstra-Test verwendet, um die Trends innerhalb der Daten zu untersuchen. Schließlich wurde $r$ verwendet, um die Intensität der Beziehung zwischen den Variablen zu messen (Rosenthal 1991, S. 19). Cohen schlägt vor, dass die Größen für die Wirkungen klein $(0,1)$, mittel $(0,3)$ oder groß $(0,5)$ einzuordnen sind. In den nächsten Abschnitten werden folgende Abkürzungen verwendet: $H$ entspricht der Kruskal-WallisStatistik, $U$ repräsentiert die MannWhitney-U-Statistik, während $J$ die beobachtete $J-T$-Statistik symbolisiert.

\subsubsection{Implementierte Prozesse und Reifegrad (T1)}

Die Anzahl der implementierten Prozesse für beide Versionen von ITIL, Version $2(n=248)$ und Version $3(n=193)$, wurden untersucht, um die Auswirkungen auf den Reifegrad der Implementierung $\mathrm{zu}$ verstehen. Abbildung 2 zeigt den Mittelwert und den Median der Zahl der realisierten Prozesse für die ITIL-Version 2 oder ITIL-Version 3 für jeden der einzelnen Reifegrade.

Im Allgemeinen beeinflusst die Zahl der implementierten Prozesse den Reifegrad der Umsetzung signifikant (Version $2 H(4)=99,03, p<0,001$, Version $3 H(4)=82,108, p<0,001)$. Es wurden Mann-Whitney-U-Tests durchgeführt, um die Ergebnisse weiter zu untersuchen. Tabelle 5 zeigt, dass die Anzahl der implementierten Prozesse beim Vergleiche der Stufen anfänglich (1) und definiert (3) signifikant ist. Die gleiche Situation lässt sich beim Vergleich der Stufen anfänglich (1) und optimiert (5) feststellen. Beim Vergleich des Grades definiert (3) mit dem Grad optimiert (5) wurde ebenfalls Signifikanz beobachtet. Mithilfe des Benchmarks von Cohen zeigt sich, dass es eine mittlere bis große Veränderung der Anzahl der implementierten Prozesse gibt, wenn die Reife zunimmt.

Mithilfe des Jonckheere-Tests kann ein signifikanter Trend in den Daten beobachtet werden. Wenn der Grad der Reife steigt, steigt auch der Median der Anzahl der implementierten Prozesse (Version 2: $J=18001, z=10,49, r=0,67$, Version 3: $J=11398, z=9,63, r=0,69$ ).

Im Ergebnis kann festgehalten werden, dass unabhängig von der jeweiligen ITIL-Version die Anzahl der implementierten Prozesse mit dem Grad der Reife der Implementierung steigt.

\subsubsection{Wahrgenommene Herausforderungen und Reifegrad (T2)}

Die Befragten wurden gebeten, Herausforderungen auf einer Skala von 1 bis 5 einzuordnen, wobei 1 keine Herausforderung und 5 große Herausforderung bedeutete. Abbilding 3 zeigt die wahrgenommene Reihenfolge der Herausforderungen. Die am niedrigsten eingeordnete Herausforderung war das Fehlen von Wissen und Fähigkeiten, sehr dicht gefolgt von der Unterstützung durch die Unternehmensführung. Eine Herausforderung, die von den Befragten als sehr hoch eingestuft wurde, ist das Fehlen von Ressourcen.

Tabellen 6 und 7 zeigen die Mittelwerte und Mediane für jede Herausforderung und den Reifegrad, während Abb. 4 und 5 eine graphische Darstellung der durchschnittlichen Bewertung der Wahrnehmung der Herausforderung für jede der untersuchten Herausforderungen für Unternehmen zu verschiedenen wahrgenommenen Reifegrade darstellen. Hier kann ein Abwärtstrend beobachtet werden. Sobald der wahrgenommene Reifegrad steigt, nimmt die Wahrnehmung der Herausforderung der Umsetzung ab.

Im Allgemeinen sind Herausforderungen der Einführung von ITIL wesentlich durch die Reife der ITIL-Implementierung geprägt: Mangelnde Unterstützung durch die Geschäftsleitung $(H(4)=37,75$, $p<0,001)$, Geschäftliches Verständnis der Ziele von ITIL $(H(4)=$ 

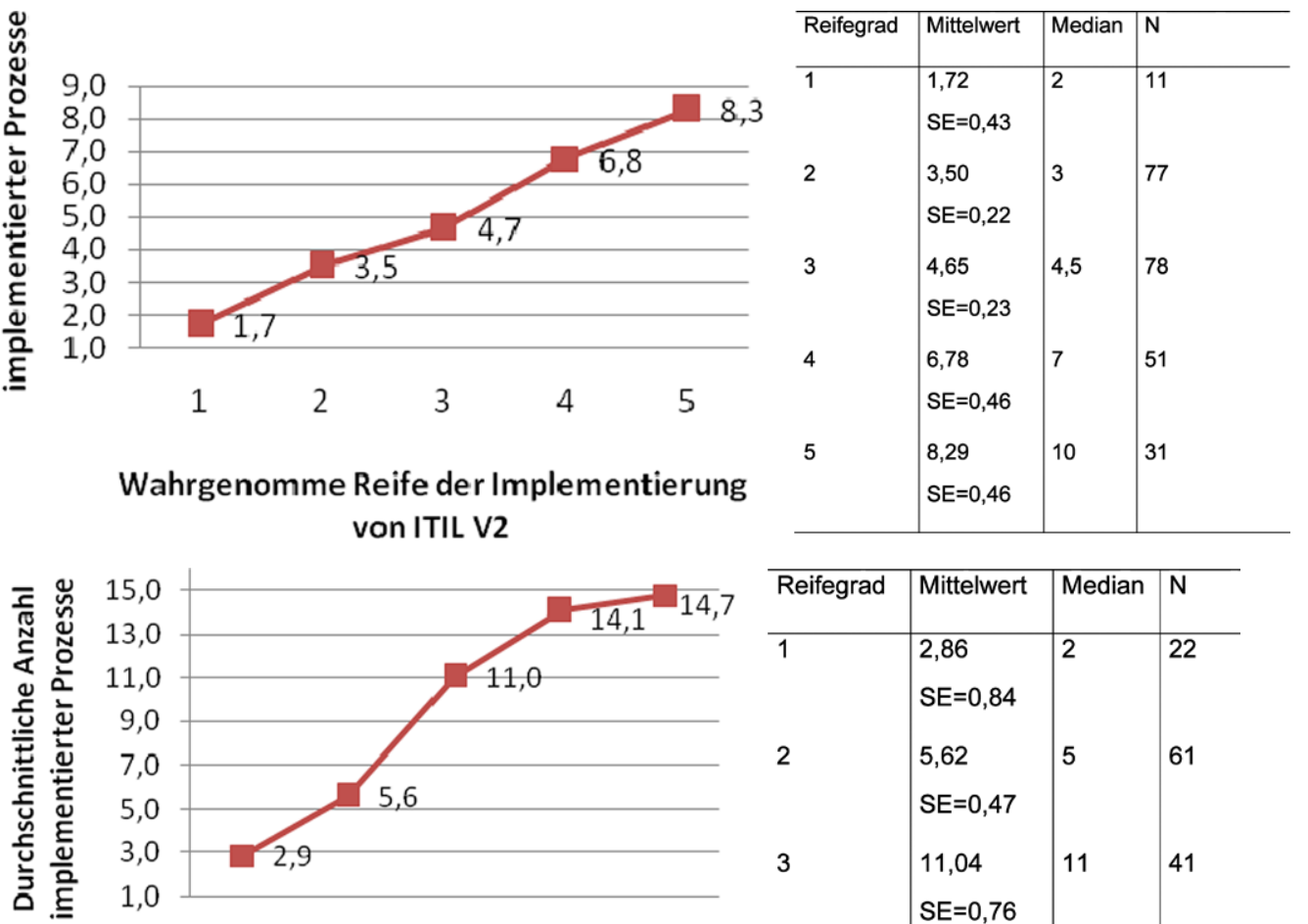

Wahrgenomme Reife der Implementierung von ITIL V2

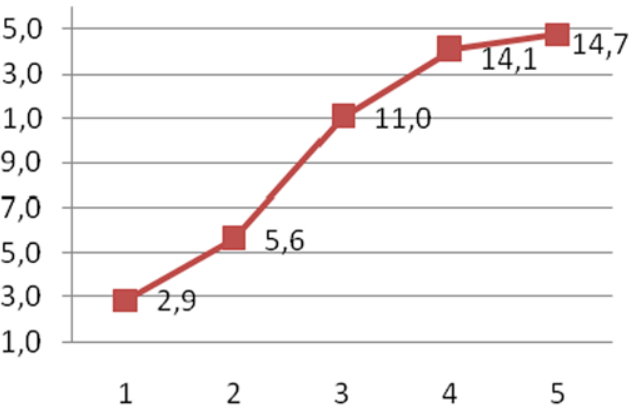

Wahrgenomme Reife der Implementierung von ITIL V3

\begin{tabular}{l|l|l|l}
\hline Reifegrad & Mittelwert & Median & $\mathrm{N}$ \\
\hline 1 & $\begin{array}{l}2,86 \\
\mathrm{SE}=0,84\end{array}$ & 2 & 22 \\
2 & $\begin{array}{l}5,62 \\
\mathrm{SE}=0,47\end{array}$ & 5 & 61 \\
3 & $\begin{array}{l}11,04 \\
\mathrm{SE}=0,76\end{array}$ & 11 & 41 \\
4 & 14,07 & 14 & 43 \\
& $\mathrm{SE}=0,84$ & &
\end{tabular}

Abb. 2 Deskriptive Statistiken der implementierten Prozesse für Version 2 und 3

Tab. 5 Ergebnisse des Mann-Whitney-Tests für ITIL-Versionen und Reifegrad

\begin{tabular}{|c|c|c|c|c|c|c|c|c|c|}
\hline & \multicolumn{3}{|c|}{ Grad 1 verglichen mit Grad 3} & \multicolumn{3}{|c|}{ Grad 1 verglichen mit Grad 5} & \multicolumn{3}{|c|}{ Grad 3 verglichen mit Grad 5} \\
\hline & $U$ & $p$ & $r$ & $U$ & $p$ & $r$ & $U$ & $p$ & $r$ \\
\hline ITIL Version 2 & 98,5 & $0,000^{\mathrm{a}}$ & $-0,44$ & 12,0 & $0,000^{\mathrm{a}}$ & $-0,73$ & 323,5 & $0,000^{\mathrm{a}}$ & $-0,58$ \\
\hline ITIL Version 3 & 75,5 & $0,000^{\mathrm{a}}$ & $-0,68$ & 83,0 & $0,000^{\mathrm{a}}$ & $-0,61$ & 345,0 & $0,013^{\mathrm{a}}$ & $-0,30$ \\
\hline
\end{tabular}

a Signifikanz bei 0,0167

Abb. 3 Deskriptive Statistik für die Bewertung der Herausforderungen ( $1=$ keine Herausforderung, $5=$ große Herausforderung)

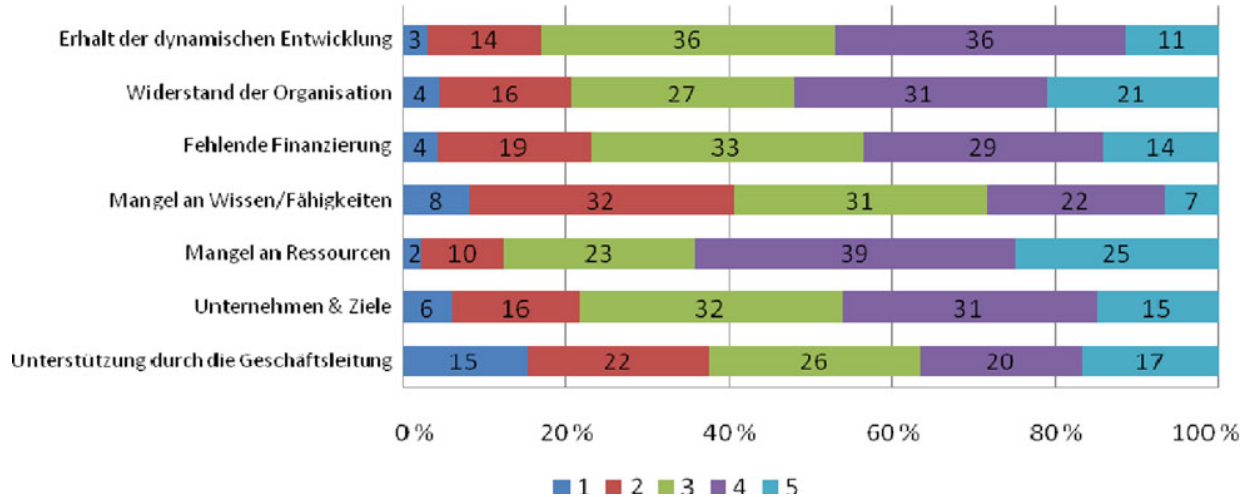

$42,19, \quad p<0,001)$, Mangel an Ressourcen, Zeit oder Personen $(H(4)=$ 32,39, $p<0,001)$, Mangel an internen Kenntnissen und Fähigkeiten in Bezug auf ITIL $(H(4)=$ 42,86, $\quad p<0,001)$, Fehlende Fi-
nanzierung/Kosten der Einführung $(H(4)=14,21, \quad p<0,01)$, Organisatorischer/Kultureller Widerstand gegen Veränderungen $(H(4)=$ $33,41, p<0,001)$, Erhalt der dynamischen Entwicklung/Fortschritt stagniert
$(H(4)=18,88, p<0,005)$. Auch im Anschluss an diese Ergebnisse wurden Mann-Whitney-Tests durchgeführt.

Tabelle 8 stellt die Ergebnisse der selektiven Vergleiche dar. Es kann beobachtet 
Tab. 6 Deskriptive Statistik zu jedem Reifegrad für jede Herausforderung (Teil I)

\begin{tabular}{|c|c|c|c|c|c|c|c|c|c|c|}
\hline \multirow[t]{2}{*}{ Reifegrad } & \multirow[t]{2}{*}{$N$} & \multicolumn{3}{|c|}{$\begin{array}{l}\text { Unterstützung durch die } \\
\text { Geschäftsleitung }\end{array}$} & \multicolumn{3}{|c|}{$\begin{array}{l}\text { Verständnis der Ziele von ITIL im } \\
\text { Geschäftsbereich }\end{array}$} & \multicolumn{3}{|c|}{$\begin{array}{l}\text { Mangel an Ressourcen } \\
\text { (Zeit oder Personen) }\end{array}$} \\
\hline & & $\bar{M}$ & $\mathrm{SE}$ & $\mathrm{Mdn}$ & $\bar{M}$ & SE & $\mathrm{Mdn}$ & $\bar{M}$ & SE & Mdn \\
\hline 1 & 62 & 3,26 & 0,15 & 3 & 3,79 & 0,13 & 4 & 4,15 & 0,12 & 4 \\
\hline 2 & 153 & 3,38 & 0,11 & 3 & 3,57 & 0,09 & 4 & 3,89 & 0,08 & 4 \\
\hline 3 & 123 & 3,01 & 0,11 & 3 & 3,33 & 0,09 & 3 & 3,74 & 0,07 & 4 \\
\hline 4 & 95 & 2,63 & 0,12 & 2 & 2,99 & 0,10 & 3 & 3,57 & 0,11 & 4 \\
\hline 5 & 58 & 2,34 & 0,19 & 2 & 2,79 & 0,17 & 3 & 3,22 & 0,13 & 3 \\
\hline
\end{tabular}

Tab. 7 Deskriptive Statistik zu jedem Reifegrad für jede Herausforderung (Teil II)

\begin{tabular}{|c|c|c|c|c|c|c|c|c|c|c|c|c|c|}
\hline \multirow[t]{2}{*}{ Reifegrad } & \multirow[t]{2}{*}{$N$} & \multicolumn{3}{|c|}{$\begin{array}{l}\text { Mangel an } \\
\text { Wissen/Fähigkeiten in } \\
\text { Bezug auf ITIL }\end{array}$} & \multicolumn{3}{|c|}{$\begin{array}{l}\text { Fehlende } \\
\text { Finanzierung/Kosten } \\
\text { der Einführung } \\
\end{array}$} & \multicolumn{3}{|c|}{$\begin{array}{l}\text { Organisatorischer/Kultureller } \\
\text { Widerstand gegen } \\
\text { Veränderungen }\end{array}$} & \multicolumn{3}{|c|}{$\begin{array}{l}\text { Erhalt der dynamischen } \\
\text { Entwicklung/Fortschritt } \\
\text { stagniert }\end{array}$} \\
\hline & & $\bar{M}$ & SE & $\overline{M d n}$ & $\mathrm{M}$ & SE & $\overline{M d n}$ & $\mathrm{M}$ & SE & Mdn & $\mathrm{M}$ & SE & $\overline{M d n}$ \\
\hline 1 & 62 & 3,45 & 0,15 & 4 & 3,52 & 0,13 & 3 & 3,89 & 0,12 & 4 & 3,63 & 0,11 & 4 \\
\hline 2 & 153 & 3,10 & 0,08 & 4 & 3,41 & 0,08 & 4 & 3,74 & 0,09 & 4 & 3,50 & 0,07 & 4 \\
\hline 3 & 123 & 2,70 & 0,08 & 3 & 3,35 & 0,09 & 3 & 3,39 & 0,10 & 3 & 3,45 & 0,09 & 4 \\
\hline 4 & 95 & 2,60 & 0,09 & 3 & 3,20 & 0,11 & 3 & 3,25 & 0,11 & 3 & 3,13 & 0,10 & 3 \\
\hline 5 & 58 & 2,41 & 0,14 & 2 & 2,88 & 0,14 & 3 & 2,93 & 0,16 & 3 & 3,10 & 0,12 & 3 \\
\hline
\end{tabular}

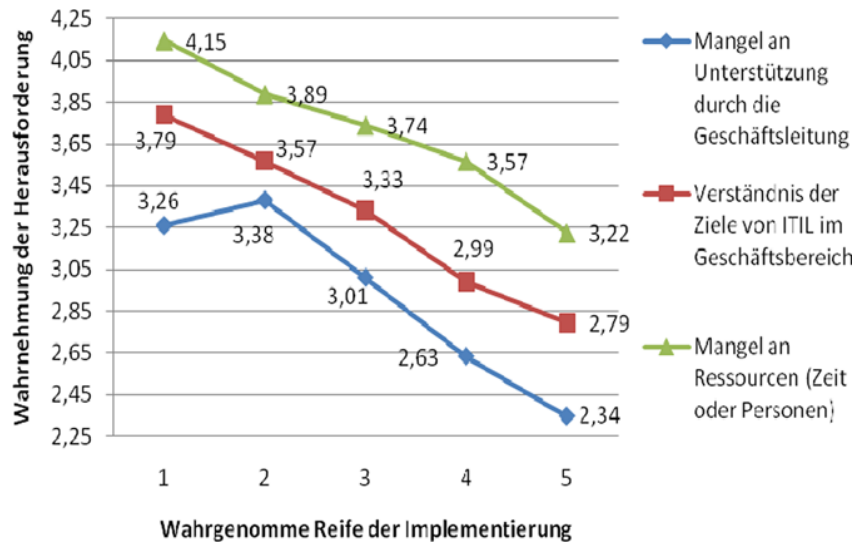

Abb. 4 Deskriptive Statistik zu jedem Reifegrad für jede Herausforderung (Teil I)

werden, dass zwischen den Ebenen anfänglich (1) und definiert (3) Signifikanz für ein Verständnis der Ziele von ITIL im Geschäftsbereich, den Mangel an Ressourcen, Zeit oder Personen, den Mangel an internen Kenntnissen und Fähigkeiten in Bezug auf ITIL sowie einen organisatorischen bzw. kulturellen Widerstand gegen Veränderungen besteht. Basierend auf dem Benchmark von Cohen kann eine kleine bis mittlere Veränderung bei der Reduzierung der Wahrnehmung der Herausforderungen festgestellt werden, sobald die sich die Reife der Implementierung erhöht. Es konnte jedoch keine Signifikanz für die mangelnde Unterstützung durch die
Geschäftsleitung, fehlende Finanzierung/Kosten der Einführung und den Erhalt der dynamischen Entwicklung/ Fortschritt stagniert festgestellt werden.

Beim Vergleich von anfänglich (1) mit optimiert (5) kann Signifikanz für alle Variablen mit einer mittleren Effektstärke beobachtet werden.

Schließlich kann Signifikanz beim Vergleich von Reifegrad definiert (3) und Reifegrad optimiert (5) für fehlende Unterstützung durch die Geschäftsleitung, geschäftliches Verständnis der Ziele von ITIL, Mangel an Ressourcen, Zeit oder Personen, Fehlende Finanzierung/Kosten der Einführung, Organisatorischer/Kultureller Widerstand ge- gen Veränderungen festgestellt werden. Marginale Signifikanz besteht bei den Herausforderungen Mangel an internen Kenntnissen und Fähigkeiten in Bezug auf ITIL und Erhalt der dynamischen Entwicklung/Fortschritt stagniert.

Der Jonckheere-Test ergab einen signifikanten Trend in den Daten. Wenn der Grad der Reife steigt, nimmt der Median der Herausforderungen ab (Mangel an Unterstützung durch die Geschäftsleitung $J=36500, \quad z=-5,9$, $r=-0,26$, Geschäftliches Verständnis der Ziele von ITIL $J=35298, z=-6,6$, $r=-0,30$ Mangel an Ressourcen, Zeit oder Personen $J=37332, \quad z=-5,5$, $r=-0,25$, Mangelnde interne Kenntnisse und Fähigkeiten in Bezug auf ITIL $J=35233, z=-6,7, \quad r=-0,30$, Fehlende Finanzierung/Kosten der Einführung $J=40536, z=-3,6, \quad r=-0,16$, Organisatorischer/Kultureller Widerstand gegen Veränderungen $J=36699$, $z=-5,8, r=-0,26$, Erhalt der dynamischen Entwicklung/Fortschritt stagniert $J=39816, z=-4.0, r=-0,18$ ).

Zusammenfassend lässt sich feststellen, dass sich die Reife der Implementierung erhöht, wenn die Wahrnehmung der Schwierigkeiten bei der Bewältigung der im Rahmen dieser Forschung untersuchten Herausforderungen abnimmt. Die Herausforderung „Erhalt der dynamischen Entwicklung/Fortschritt 
Tab. 8 Ergebnisse des Mann-Whitney-Tests für die Wahrnehmung der Herausforderungen und Reifegrade

\begin{tabular}{|c|c|c|c|c|c|c|c|c|c|}
\hline \multirow[t]{2}{*}{ Herausforderungen } & \multicolumn{3}{|c|}{ Grad 1 verglichen mit Grad 3} & \multicolumn{3}{|c|}{ Grad 1 verglichen mit Grad 5} & \multicolumn{3}{|c|}{ Grad 3 verglichen mit Grad 5} \\
\hline & $\bar{U}$ & $p$ & $r$ & $\bar{U}$ & $p$ & $R$ & $\bar{U}$ & $p$ & $r$ \\
\hline $\begin{array}{l}\text { Mangel an Unterstützung durch } \\
\text { die Geschäftsleitung }\end{array}$ & 3375,0 & 0,189 & $-0,10$ & 1104,5 & $0,000^{\mathrm{a}}$ & $-0,34$ & 2492,0 & $0,001^{\mathrm{a}}$ & $-0,25$ \\
\hline $\begin{array}{l}\text { Verständnis der Ziele von ITIL im } \\
\text { Geschäftsbereich }\end{array}$ & 2736,5 & $0,001^{\mathrm{a}}$ & $-0,24$ & 993,0 & $0,000^{\mathrm{a}}$ & $-0,40$ & 2634,0 & $0,003^{\mathrm{a}}$ & $-0,22$ \\
\hline $\begin{array}{l}\text { Mangel an Ressourcen (Zeit oder } \\
\text { Personen) }\end{array}$ & 2756,5 & $0,001^{\mathrm{a}}$ & $-0,24$ & 894,0 & $0,000^{\mathrm{a}}$ & $-0,45$ & 2496,5 & $0,001^{\mathrm{a}}$ & $-0,26$ \\
\hline $\begin{array}{l}\text { Mangelnde internen } \\
\text { Kenntnissen/Fähigkeiten Bezug } \\
\text { auf ITIL }\end{array}$ & 2461,0 & $0,000^{\mathrm{a}}$ & $-0,30$ & 977,5 & $0,000^{\mathrm{a}}$ & $-0,40$ & 2884,5 & 0,031 & $-0,16$ \\
\hline $\begin{array}{l}\text { Fehlende Finanzierung/Kosten } \\
\text { der Einführung }\end{array}$ & 3433,0 & 0,254 & $-0,09$ & 1234,0 & $0,002^{\mathrm{a}}$ & $-0,28$ & 2724,5 & $0,008^{\mathrm{a}}$ & $-0,20$ \\
\hline $\begin{array}{l}\text { Organisatorischer/Kultureller } \\
\text { Widerstand gegen Veränderungen }\end{array}$ & 2825,0 & $0,003^{\mathrm{a}}$ & $-0,22$ & 1017,5 & $0,000^{\mathrm{a}}$ & $-0,39$ & 2785,0 & $0,014^{\mathrm{a}}$ & $-0,18$ \\
\hline $\begin{array}{l}\text { Erhalt der dynamischen } \\
\text { Entwicklung/Fortschritt stagniert }\end{array}$ & 3419,5 & 0,225 & $-0,09$ & 1245,0 & $0,003^{\mathrm{a}}$ & $-0,28$ & 2869,0 & $0,027^{\mathrm{a}}$ & $-0,17$ \\
\hline
\end{tabular}

${ }^{\text {a }}$ Signifikanz bei 0,0167

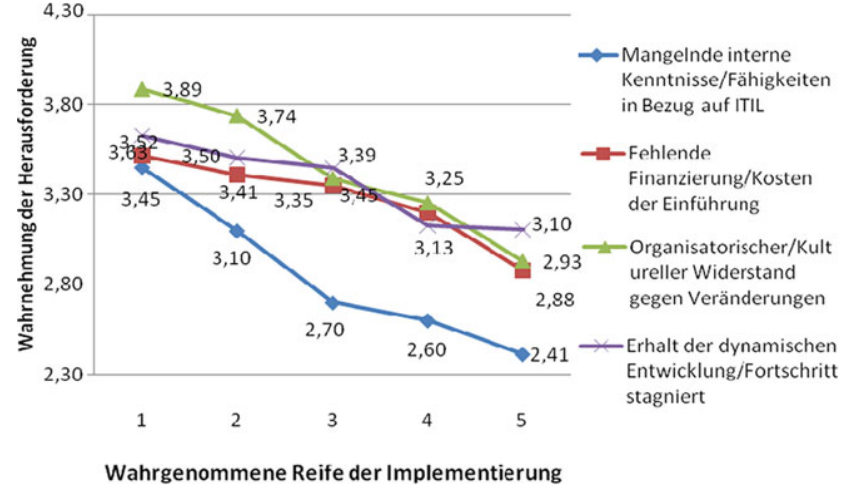

Abb. 5 Deskriptive Statistiken zu jedem Reifegrad für jede Herausforderung (Teil II)

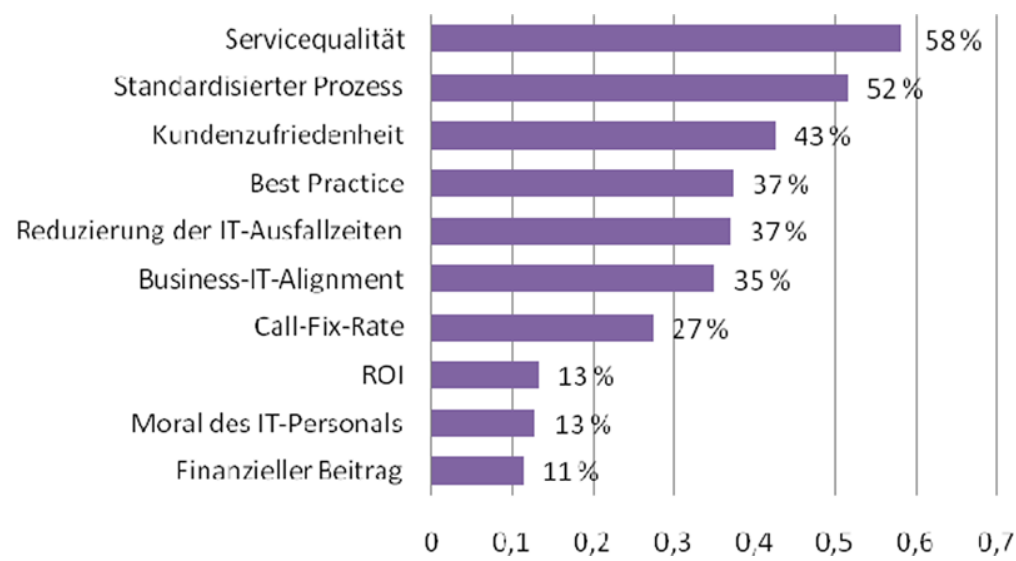

Abb. 6 Anteile der Unternehmen, die einen Vorteil realisiert haben

stagniert" ist der einzige Faktor, der keine Signifikanz bei der Analyse der Reifegrade anfänglich (1) und definiert (3) aufweist und nur marginale Signifikanz beim Vergleich von definiert (3) und optimiert (5) besitzt. Allerdings besteht bei der Überprüfung von anfänglich (1) und optimiert (5) Signifikanz. Bei der Analyse der Variablen Mangel an Unterstützung durch die Geschäftsleitung und Fehlende Finanzierung/Kosten der Einführung kann beobachtet werden, dass auf den unteren Ebenen der Implementierung keine Signifikanz besteht. Vergleicht man jedoch definiert (3) mit optimiert (5), kann Signifikanz festgestellt werden.

\subsubsection{Zahl der realisierten Vorteile und Reifegrade (T3)}

Abbildung 6 stellt die Vorteile der Unternehmen, die ein ITSM-Framework implementiert haben, im Detail dar. Insgesamt scheint die Abb. 6 die Annahme nahe zu legen, dass die Hälfte der Befragten eine Verbesserung der Servicequalität sowie eine Zunahme der Verwendung standardisierter Prozessen wahrnimmt. Drei Vorteile, die nur von knapp von einem Zehntel der Befragten angegeben wurden, umfassen den Return on Investment (ROI), die Moral des ITPersonals sowie die Verbesserung der Messung des finanziellen Beitrags der IT.

Bei der Durchführung des KruskalWallis-Tests wird die Zahl der realisierten Vorteile signifikant durch den Reifegrad der Implementierung beeinflusst $(H(4)=134,49, \quad p<0,001$ für die Gesamtzahl der realisierten Vorteile, $H(4)=139,37, p<0,001$ für die realisierte Vorteile, die durch Metriken unterstützt werden, $H(4)=91,64$, $p<0,001$ für realisierte Vorteile, die vom Geschäftsbereich wahrgenommen wirden). Der Mittelwert und der Median der 
Tab. 9 Deskriptive Statistik der realisierten Vorteile $(n=491)$

\begin{tabular}{|c|c|c|c|c|c|c|c|c|c|c|}
\hline \multirow[t]{2}{*}{ Reifegrad } & \multirow[t]{2}{*}{$N$} & \multicolumn{3}{|c|}{ Gesamtzahl realisierter Vorteile } & \multicolumn{3}{|c|}{$\begin{array}{l}\text { Gesamtzahl realisierter } \\
\text { Vorteile, die durch Metriken } \\
\text { unterstützt werden }\end{array}$} & \multicolumn{3}{|c|}{$\begin{array}{l}\text { Gesamtzahl realisierter Vorteile, } \\
\text { die vom Geschäftsbereich } \\
\text { wahrgenommen wurden }\end{array}$} \\
\hline & & M & SE & Mdn & M & SE & Mdn & M & SE & Mdn \\
\hline 1 & 62 & 1,03 & 0,19 & 0 & 0,48 & 0,15 & 0 & 0,44 & 0,14 & 0 \\
\hline 2 & 153 & 2,46 & 0,14 & 2 & 1,04 & 0,1 & 1 & 0,75 & 0,1 & 0 \\
\hline 3 & 123 & 4,06 & 0,19 & 4 & 2,49 & 0,17 & 2 & 1,71 & 0,16 & 1 \\
\hline 4 & 95 & 4,13 & 0,21 & 4 & 2,65 & 0,19 & 2 & 2,23 & 0,22 & 2 \\
\hline 5 & 58 & 4,89 & 0,37 & 5 & 3,89 & 0,37 & 4 & 3,34 & 0,41 & 3 \\
\hline
\end{tabular}

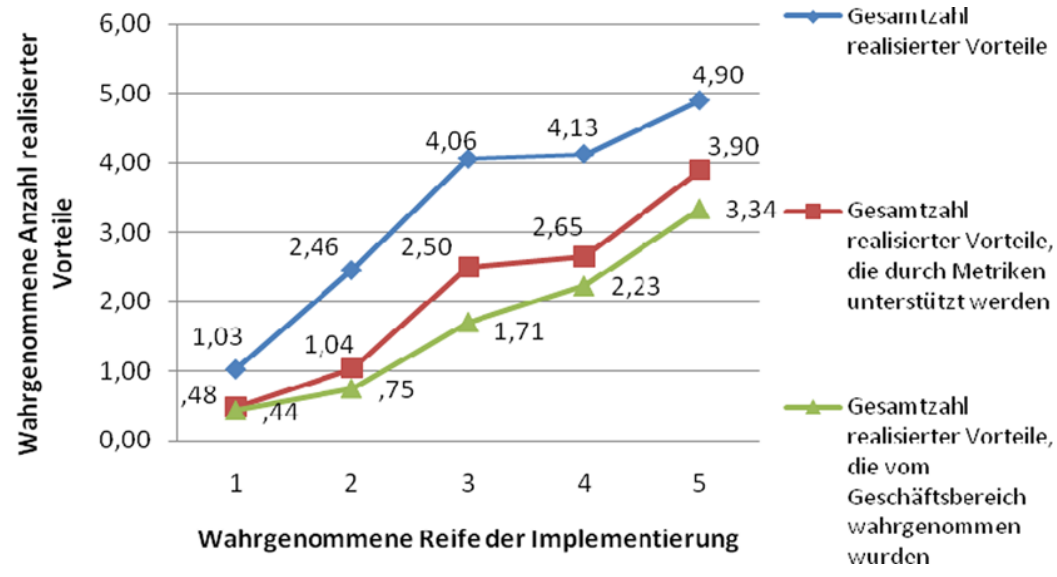

Abb. 7 Deskriptive Statistik der realisierten Vorteile $(n=491)$

Vorteile für jeden der verschiedenen Reifegrade werden in Tab. 9 dargestellt.

Abbildung 7 stellt die wahrgenommene Anzahl der realisierten Vorteile für Unternehmen mit verschiedenen Reifegraden der Umsetzung dar. In Abb. 7 ist ein Aufwärtstrend $\mathrm{zu}$ beobachten. Unternehmen, die sich auf höheren Ebenen der wahrgenommenen Implementierungsreife befinden, glauben, eine gröBere Anzahl von Vorteilen zu realisieren.

Wie in Tab. 10 gezeigt, ist die Zahl der realisierten Vorteile signifikant höher bei einem Vergleich des Reifegrads anfänglich (1) mit dem Reifegrad definiert (3). Basierend auf dem Benchmark von Cohen liegt eine große Veränderung der Zahl der realisierten Vorteile vor, sobald die Reife zunimmt. Dies lässt sich auch beobachten beim Vergleich der Stufe anfänglich (1) mit der Stufe optimiert (5). Schließlich lässt sich beim Vergleich der Grade definiert (3) und optimiert (5) keine Signifikanz feststellen. Dennoch kann Signifikanz bei der Untersuchung der Zahl der realisierten Vorteile, die durch Metriken unterstützt wurden sowie bei der Analyse der Zahl der realisierten Vorteile, die von den Geschäftsbereichen wahrgenommen wurden, festgestellt werden.

Zur Untermauerung der Ergebnisse wurde eine zusätzliche Analyse für jeden der aufgeführten Vorteile durchgeführt. In dieser Analyse werden die individuellen Vorteile der Realisierung auf den verschiedenen Reifestufen ausgewertet. Jede Variable ist dichotom (realisierter Vorteil oder nicht realisierter Vorteil). Der Kruskal-Wallis-Test zeigt, dass alle realisierten Vorteile signifikant durch den Grad der Implementierungsreife beeinflusst werden (Servicequalität $H(4)=92,08, p<0,001$, Kundenzufriedenheit $H(4)=44,41, p<0,001$, Ausfallzeit von IT-Ressourcen $H(4)=46,41$, $p<0,001$, Vorteile durch die Nutzung einer Best Practice $H(4)=42,05$, $p<0,001, \quad$ Business-IT-Alignment $H(4)=25,45, p<0,001$, Call-Fix-Rate $H(4)=34,65, p<0,001$, standardisierte Prozesse $H(4)=68,20, p<0,001$, IT-Moral $H(4)=19,61, \quad p<0,001$, Return on Investment $H(4)=26,40$, $p<0,001$, Messung des finanziellen Beitrags $H(4)=18,93, \quad p<0,001)$. Mann-Whitney-Tests wurden im Nachgang zu den Ergebnissen durchgeführt.

Tabelle 11 enthält die Ergebnisse aus diesem Test. Beim Vergleich der Unter- nehmen auf der Ebene anfänglich (1) mit denen der Ebene definiert (3) wiesen alle Variablen eine signifikante Veränderung auf, mit Ausnahme der Verbesserung der IT-Moral, des Return on Investment und des finanziellen Beitrags. Beim Vergleich der Ebenen definiert (3) und optimiert (5) war nur der Return on Investment signifikant, alle anderen Variablen wiesen keine signifikante Veränderung auf. Dies bestätigt und erweitert die Ergebnisse der vorangegangenen Analyse (T3a). Schließlich kann bei der Gegenüberstellung der Vorteile auf den Ebenen anfänglich (1) und optimiert (5) für alle Variablen Signifikanz beobachtet werden.

Der Jonckheere-Test zeigt einen signifikanten Trend in den Daten, da sich bei der Erhöhung des Reifegrads der Median der Zahl der realisierten Vorteile erhöht $(J=66553, z=11,44, r=0,52)$, der Median der Anzahl der realisierten Vorteile, die durch Metriken unterstützt werden, erhöht $(J=67164$, $z=11,69, r=0,54)$ und sich schließlich ebenfalls der Median der Anzahl der realisierten Vorteile erhöht, die vom Geschäftsbereich wahrgenommen wurden $(J=62730, z=9,73, r=0,44)$.

Es lässt sich zusammenfassend feststellen, dass die Zahl der realisierten Vorteile ebenfalls steigt, sobald der Grad der Reife zunimmt. Allerdings gibt es keine Zunahme in der Zahl der realisierten Vorteile zwischen den Ebenen definiert (3) und optimiert (5). Im Gegensatz dazu zeigt die Untersuchung zu späteren Stadien, dass Unternehmen in der Lage sind, die Vorteile der Implementierung von ITIL durch die verwendeten Metriken aufzuzeigen und auch die realisierten Vorteile darlegen können.

\section{Diskussion}

Die Ergebnisse der aktuellen Studie bestätigen T1. Diese These besagt, dass 
Tab. 10 Ergebnisse des Mann-Whitney-Tests für realisierte Vorteile und Reifegrade

\begin{tabular}{|c|c|c|c|c|c|c|c|c|c|}
\hline \multirow[t]{2}{*}{... pro Unternehmen } & \multicolumn{3}{|c|}{ Grad 1 verglichen mit Grad 3} & \multicolumn{3}{|c|}{ Grad 1 verglichen mit Grad 5} & \multicolumn{3}{|c|}{ Grad 3 verglichen mit Grad 5} \\
\hline & $\bar{U}$ & $p$ & $r$ & $\bar{U}$ & $p$ & $r$ & $\bar{U}$ & $p$ & $r$ \\
\hline Gesamtzahl realisierter Vorteile & 980,5 & $0,000^{\mathrm{a}}$ & $-0,61$ & 434,0 & $0,000^{\mathrm{a}}$ & $-0,67$ & 2956,5 & 0,060 & $-0,14$ \\
\hline $\begin{array}{l}\text { Gesamtzahl realisierter Vorteile, die } \\
\text { durch Metriken unterstützt werden }\end{array}$ & 1209,5 & $0,000^{\mathrm{a}}$ & $-0,57$ & 478,5 & $0,000^{\mathrm{a}}$ & $-0,67$ & 2529,5 & $0,002^{\mathrm{a}}$ & $-0,24$ \\
\hline $\begin{array}{l}\text { Gesamtzahl realisierter Vorteile, die } \\
\text { vom Geschäftsbereich wahrgenommen } \\
\text { wurden }\end{array}$ & 2099,0 & $0,000^{\mathrm{a}}$ & $-0,40$ & 658,0 & $0,000^{\mathrm{a}}$ & $-0,59$ & 2511,5 & $0,001^{\mathrm{a}}$ & $-0,24$ \\
\hline
\end{tabular}

a Signifikanz bei 0,0167

Tab. 11 Ergebnisse des Mann-Whitney-Tests für die einzelnen Vorteile und Reifegrade

\begin{tabular}{|c|c|c|c|c|c|c|c|c|c|}
\hline \multirow[t]{2}{*}{ Verbesserungen von } & \multicolumn{3}{|c|}{ Grad 1 verglichen mit Grad 3} & \multicolumn{3}{|c|}{ Grad 1 verglichen mit Grad 5} & \multicolumn{3}{|c|}{ Grad 3 verglichen mit Grad 5} \\
\hline & $U$ & $p$ & $r$ & $U$ & $p$ & $r$ & $U$ & $p$ & $r$ \\
\hline Servicequalität & 1544,0 & $0,000^{\mathrm{a}}$ & $-0,57$ & 720,0 & $0,000^{\mathrm{a}}$ & $-0,59$ & 3551,0 & 0,560 & $-0,05$ \\
\hline Kundenzufriedenheit & 2536,5 & $0,000^{\mathrm{a}}$ & $-0,32$ & 908,0 & $0,000^{\mathrm{a}}$ & $-0,49$ & 2994,5 & 0,027 & $-0,15$ \\
\hline Reduzierung der IT-Ausfallzeiten & 2416,0 & $0,000^{\mathrm{a}}$ & $-0,37$ & 1046,0 & $0,000^{\mathrm{a}}$ & $-0,47$ & 3382,0 & 0,301 & $-0,05$ \\
\hline Best Practice & 2352,5 & $0,000^{\mathrm{a}}$ & $-0,37$ & 978,0 & $0,000^{\mathrm{a}}$ & $-0,48$ & 3306,5 & 0,218 & $-0,07$ \\
\hline Business-IT-Alignment & 2909,0 & $0,001^{\mathrm{a}}$ & $-0,24$ & 1220,0 & $0,000^{\mathrm{a}}$ & $-0,34$ & 3266,0 & 0,178 & $-0,08$ \\
\hline Call-Fix-Rate & 2601,5 & $0,000^{\mathrm{a}}$ & $-0,33$ & 1137,0 & $0,000^{\mathrm{a}}$ & $-0,41$ & 3389,0 & 0,311 & $-0,05$ \\
\hline Standardisierter Prozess & 1856,0 & $0,000^{\mathrm{a}}$ & $-0,48$ & 852,0 & $0,000^{\mathrm{a}}$ & $-0,53$ & 3521,0 & 0,494 & $-0,01$ \\
\hline Moral des IT-Personals & 3346,0 & 0,018 & $-0,16$ & 1449,0 & $0,003^{\mathrm{a}}$ & $-0,26$ & 3311,5 & 0,176 & $-0,08$ \\
\hline ROI & 3502,5 & 0,034 & $-0,15$ & 1300,0 & $0,000^{\mathrm{a}}$ & $-0,38$ & 2869,5 & $0,001^{\mathrm{a}}$ & $-0,24$ \\
\hline Finanzieller Beitrag & 3533,0 & 0,073 & $-0,13$ & 1422,0 & $0,001^{\mathrm{a}}$ & $-0,30$ & 3083,0 & 0,018 & $-0,17$ \\
\hline
\end{tabular}

${ }^{\text {a }}$ Signifikanz bei 0,0167

die wahrgenommene Reife der ITILImplementierung ansteigt, wenn mehr Prozesse von ITIL implementiert werden. Es kann ein positiver direkter $\mathrm{Zu}$ sammenhang zwischen der Anzahl der implementierten ITIL-Prozesse und dem Reifegrad der ITIL-Implementierung beobachtet werden. Dieses Ergebnis kann auch einen Einblick darüber geben, wie die Unternehmen im Rahmen der Einführung von ITIL die ITIL-Prozesse umsetzen, wobei eher eine Steigerung der Implementierung von Prozessen anstatt der Umsetzung aller Prozesse auf einmal stattfindet. Während ITFührungskräfte die zu implementierenden Prozesse manuell auswählen, verstehen sie das Framework als Ganzes und nehmen die Reife ihrer Implementierung aus der Perspektive der Reife des gesamten Frameworks wahr.

Im Allgemeinen wird die zweite These T2 ebenfalls bestätigt. Diese besagt, dass die Wahrnehmung der aufgeführten Herausforderungen der Implementierung abnimmt, wenn die Reifegrade der Implementierung steigen. Dies kann als Resultat der Tatsache verstanden werden, dass die Unternehmen, die im Rahmen der Einführung die ersten Herausforderungen der Umsetzung überwinden, Erfahrungen sammeln und die Herausforderungen der Zukunft als weniger komplex wahrnehmen als solche auf den früheren Ebenen. Des Weiteren folgt diese Beobachtung dem Muster des Lernkurvenmodells, bei dem auf den ersten Ebenen Schwierigkeiten bei der Umsetzung durchlaufen werden, die jedoch durch gewonnene Erfahrung verringert werden können.

Dies kann auch bedeuten, dass diese Herausforderungen reduziert werden, da die Vorteile von ITIL für das Unternehmen und für die im Implementierungsprojekt beteiligten Personen aufgezeigt wurden. Wie Huber (1991) erklärt, tritt organisationales Lernen in einem größeren Ausmaß auf, wenn die gewonnenen Erkenntnisse als nützlich anerkannt werden. Mit anderen Worten wird die Organisation eher lernen, wenn den einzelnen Mitarbeitern und der Organisation die Vorteile be- wusst werden. Im Falle dieser Studie kann der Grund für die Reduzierung der Wahrnehmung für die Herausforderungen mit der Zeit auch in den durch die Implementierung erreichten Vorteilen liegen, die die Organisation und die Personen dazu ermutigt, zu lernen und weitere Prozesse zu implementieren.

Bei genauerer Betrachtung zeigen die Herausforderungen wie „Mangel an Unterstützung durch die Geschäftsleitung, fehlende Finanzierung und Erhalt der Dynamik/Projekt stagniert" keine Signifikanz bei einem Vergleich der Reifegrade der Implementierung anfänglich (1) und definiert (3). Vergleicht man jedoch die Stadien definiert (3) und optimiert (5), sinkt die Wahrnehmung dieser Herausforderungen. Dies kann daraus resultieren, dass in den früheren Reifegraden noch Skepsis vorherrscht. Umgekehrt werden Unternehmen in den späteren Stadien der Implementierung, sobald die Vorteile der ITIL-Implementierung erkannt wurden, eher eine weitere Umsetzung von ITIL unterstützen. 
Beim Vergleich der Reifegrade definiert (3) und optimiert (5) kann marginale Signifikanz für die Herausforderungen Mangel an internen Kenntnissen und Fähigkeiten und Erhalt der Dynamik/Projekt stagniert beobachtet werden. Im Fall des Mangels an internen Kenntnisse und Fähigkeiten könnte dies durch die Tatsache begründet sein, dass die Einstellung von Personal, das spezielles Wissen in diesem Bereich hat, schwierig sein kann oder dass die Ausbildungsprogramme, die neu sind, nicht sehr häufig angeboten werden. Erhalt der Dynamik/Projekt stagniert ist die einzige Herausforderung, die nicht als statistisch signifikant in zwei der drei Vergleiche bewiesen werden konnte (Vergleich zwischen anfänglich (1) und definiert (3) sowie von definiert (3) und optimiert (5)). Dies deutet darauf hin, dass diese Herausforderung während des gesamten Projekts im Fokus von IT-Managern liegt und ein kritischer Erfolgsfaktor für die Implementierung sein könnte. Darüber hinaus kann dieser Aspekt als unabhängig von der ITIL-Implementierung betrachtet werden und könnte einen Faktor darstellen, der den Personen zuzuordnen ist, die die Implementierung von ITIL ausführen.

Schließlich erwiesen sich die Ergebnisse der Thesen T3a, T3b und T3C als statistisch signifikant. Die Tatsache, dass Vorteile aus der Einführung von ITIL resultieren stimmt mit den Ergebnissen aus den einzelnen Fallstudien über die Wirksamkeit von ITIL durch Potgieter et al. (2005) und Spremic et al. (2008) überein. Wie Hochstein et al. (2005) berichtet, stellen „quick wins“ kritische Erfolgsfaktoren bei der Umsetzung von ITIL dar. Demnach könnten einige Unternehmen versuchen, Vorteile auf den unteren Ebenen der Implementierung zu realisieren.

Wie durch die Ergebnisse von T3a beobachtet werden kann, erhöht sich die Zahl der realisierten Vorteile, sobald der Reifegrad steigt. Es besteht jedoch keine Signifikanz bei einem Vergleich der Grade definiert (3) und optimiert (5). Daher scheint mit Blick nur auf dieses Ergebnis das Gesetz vom abnehmenden Ertrag für die untersuchten Vorteile von ITIL zuzutreffen. Bei Betrachtung der individuellen Vorteile der Umsetzung zeigte keiner der untersuchten Vorteile eine Veränderung, außer der Return on Investment. Dies deutet darauf hin, dass die Unternehmen während der früheren Stadien den größten direkten Nutzen aus der ITIL-Implementierung erfahren. Es könnte auch darauf hindeuten, dass die meisten der untersuchten Variablen nur für Unternehmen in den früheren Stadien gelten und dass weitere Vorteile existieren könnten.

Betrachtet man die Ergebnisse von T3b und T3C kann nicht sicher gesagt werden, dass das Gesetz des abnehmenden Ertrags gilt. Wie aus den Ergebnissen von T3b ersichtlich wird, werden Verbesserungen, die durch Metriken unterstützt werden, auf den späteren Stufen der Implementierung wahrgenommen. Des Weiteren zeigen sich die Vorteile der ITIL-Implementierung, die von den Geschäftsbereichen wahrgenommen wurden (T3c), ebenfalls erst auf den späteren Stufen der Umsetzung.

Die Untersuchung bietet einen Einblick in die Wahrnehmung der Effektivität von ITIL, der Implementierung von Prozessen und die Entwicklung von Herausforderungen. Darüber hinaus eröffnet dieser Beitrag den Weg für zukünftige Forschungsarbeiten. In der Praxis können die Ergebnisse als Leitfaden für IT-Manager dienen, die eine Einführung von ITIL in Erwägung ziehen oder ITIL bereits eingeführt haben. Für diejenigen, die über eine Einführung nachdenken und Zweifel in Bezug auf den Nutzen von ITIL haben, zeigen die Ergebnisse, dass die Unternehmen durch die Umsetzung von ITIL eine Reihe von Vorteilen erreichen können. Für die ITAbteilungen, die ITIL bereits eingeführt haben und über die Ausweitung ihrer ITIL-Implementierung nachdenken, wird ein Überblick der Evolution von realisierbaren Vorteilen und darüber, wie die Wahrnehmung der Herausforderungen von der weiteren Implementierung von ITIL beeinflusst wird, geschaffen.

\section{Fazit}

Basierend auf den erzielten Ergebnissen in den vorangegangenen Abschnitten wurde gezeigt, dass die Anzahl der implementierten Prozesse zunimmt, wenn sich die Reife der ITIL-Implementierung erhöht. Zusätzlich konnte gezeigt werden, dass sich die Herausforderungen der Implementierung verringern, wenn sich die Reife erhöht. Dies wird anhand der Lernkurve sowie von Erkenntnissen des organisationalen Lernens erläutert. Die Ergebnisse führen zu dem Schluss, dass sich die Zahl der realisierten Vorteile erhöht, sobald die Reife von ITIL steigt. Marginaler Nutzen kann beobachtet werden, nachdem die Implementierung den Reifegrad definiert (3) erreicht. Dennoch kann in späteren Phasen der Implementierung weiterer Nutzen durch die ITIL-Implementierung erreicht werden. In diesen späteren Stadien gibt es eine Zunahme in der Nutzung von Metriken, um die Vorteile der Umsetzung zu messen sowie auch in der Anerkennung der Vorteile durch das Unternehmen.

Die Limitationen dieser Studie bestehen darin, dass sich die Untersuchung nur auf die USA und Großbritannien konzentriert und dass sie größere Unternehmen zu stark betrachtet. Diese Forschung zielte nur auf die Befragung von IT-Führungskräften und nur ihre Ansichten sind in der Studie wiedergegeben. Eine weitere Einschränkung ist, dass empirische Studien abhängig von der Qualität der von den Befragten angegebenen Daten sind. Des Weiteren nutzt der Beitrag eine wahrgenommene Reife, die auf einer einzigen Messung beruht. $\mathrm{Zu}$ dem basieren die Ergebnisse nur auf den Herausforderungen und Vorteilen, die im Rahmen der Umfrage genannt wurden.

Bei der vorliegenden Studie wurden IT-Führungskräfte befragt. Das Ziel weiterer, aktueller Untersuchungen ist daher ein besseres Verständnis durch eine Erweiterung um die Sicht der Geschäftsbereiche auf die Herausforderungen und Vorteile der ITIL-Umsetzung. Ein Vergleich der Ansichten, der Perspektive der IT und der fachlichen Sichtweise, ist hier von großer Relevanz für das Verhältnis von IT und Geschäftsbereichen und damit dem Business-ITAlignment. Zukünftige Arbeiten sollten eine Längsschnittstudie über den Einfluss von ITIL vervollständigen.

Open Access Dieser Artikel unterliegt den Bedingungen der Creative Commons Attribution Noncommercial License. Dadurch sind die nichtkommerzielle Nutzung, Verteilung und Reproduktion erlaubt, sofern der/die Originalautor/en und die Quelle angegeben sind.

\section{Anhang: Verwendete Umfrage}

(1) Which of the following statements best describes your IT organization?

- We have not adopted ITIL (Level 0)

- We are new to ITIL and have just started to implement processes (Level 1) 


\section{Zusammenfassung / Abstract}

Mauricio Marrone, Lutz M. Kolbe

\section{Einfluss von IT-Service-Management-Frameworks auf die IT-Organisation}

\section{Eine empirische Studie zu Vorteilen, Herausforderungen und Prozessen}

Über 90 Prozent aller Unternehmen nutzen IT-Service-Management-Frameworks, dennoch existiert nur wenig Forschung über deren Vorteile für IT-Abteilungen und einzelne Geschäftsbereiche. Es wurde eine internationale Befragung von 491 Unternehmen durchgeführt, um die Vorteile der IT Infrastructure Library (ITIL), dem defacto ITSM-Standard, insbesondere in Bezug auf die Entstehung dieser Vorteile zu bewerten. Darüber hinaus wurden die Wahrnehmung von Herausforderungen in Rahmen der Implementierung sowie die Anzahl der implementierten ITIL-Prozesse in Bezug auf den Fortschritt der Einführung von ITIL untersucht. Die Ergebnisse deuten darauf hin, dass die Wahrnehmung von Herausforderungen sinkt, sobald die Reife der Implementierung steigt. Die Ergebnisse zeigen darüber hinaus, dass durch eine Erhöhung der Implementierungsreife auch die Anzahl der realisierten Vorteile steigt. Des Weiteren werden die Implikationen für Praxis und Forschung diskutiert.

Schlüsselwörter: IT Infrastructure Library, IT-Service-Management, Best Practice, ITIL, ITSM, IT-Services

\section{Impact of IT Service Management Frameworks on the IT Organization}

An Empirical Study on Benefits, Challenges, and Processes

Over 90 percent of companies are estimated to use IT Service Management (ITSM) frameworks, yet there is little research on their benefits to the Information Technology (IT) department and the business units. An international survey of 491 firms was conducted to assess the benefits of the IT Infrastructure Library (ITIL), the de-facto ITSM framework, specifically on how these benefits evolve as companies increase their adoption of the ITIL model. Also studied are the perception of challenges of the implementation and the number of ITIL processes implemented in relation to the progress of the adoption of ITIL. Results indicate that as the maturity of implementation increases, the perception of challenges decreases. Findings also show that as the maturity of implementation increases, the number of realized benefits increases, as well as the number of implemented ITIL processes. Implications for practitioners and researchers are also discussed.

Keywords: IT Infrastructure Library, IT Service Management, Best Practice, ITIL, ITSM, IT Services
- We have a relatively low level of ITIL process maturity. Some processes are documented and these are generally understood, but errors are likely (Level 2)

- We have a medium level of ITIL process maturity. Processes are documented monitored for compliance (Level 3)

- We have a reasonably high level of ITIL process maturity. Our processes are documented, and measured according to established metrics (Level 4)

- We have a very high level of ITIL process maturity. Our processes are documented, understood, backed by metrics and continually reviewed for improvement (Level 5)

(2) Which version of ITIL (if any) are you using?

- ITIL V2

- ITIL V3, upgraded from V2

- ITIL V3

- Have not adopted ITIL

(The following two questions are only for respondents who answered ITIL V2 on question 2)

(3) Which of the following ITIL V2 Service Support processes have you implemented?

- Incident Management

- Problem Management

- Change Management

- Release Management

- Configuration Management

(4) Which of the following ITIL V2 Service Delivery processes have you implemented?

- Availability Management

- Capacity Management

- Financial Management

- Service Level Management

- IT Service Continuity Management

(The following five questions are only for respondents who answered ITIL V3 or ITIL V3, upgraded from V2 on question 2)

(5) Which Service Strategy process have you implemented?

- Strategy Generation

- Service Portfolio Management

- Demand Management

- Financial Management

(6) Which Service Design process have you implemented?

- Service Catalogue Management

- Service Level Management

- Availability Management

- Capacity Management 
- IT Service Continuity Management

- Information Security Management

- Supplier Management

(7) Which Service Transition process have you implemented?

- Transition Planning and Support

- Change Management

- Service Asset \& Configuration Management

- Release \& Deployment Management

- Service Validation \& Testing

- Evaluation

- Knowledge Management

(8) Which Service Operation process have you implemented?

- Incident Management

- Problem Management

- Request Fulfillment

- Event Management

- Access Management

(9) Which Continual Service Improvement process have you implemented?

- Service Level Management - Seven Level improvement process

- Service Measurement

- Service Reporting

(10) On a scale of $1-5$, where $1=$ No Challenge and $5=$ Major Challenge, how would you rate the following barriers to ITIL implementation in your organization?

- Lack of Executive sponsorship

- Business understanding of ITIL objectives

- Lack of resources (time or people)

- Lack of internal knowledge/skills relating to ITIL

- Lack of funding/cost of adoption

- Organization/cultural resistance to change

- Maintaining

momentum/progress stagnates

(11) Owing to the ITIL implementation, have you had an improvement in the following areas?

- Service Quality

- Customer satisfaction

- Standardized process adoption across all of IT

- Interaction of IT with rest of business

- Reduction in IT downtime

- Return on investments in IT

- Benefited from best practice experience of others
- Financial contribution of IT to the business

- Call fix rate

- Morale of IT staff

(Follow-up question, only for the selected elements of the previous question)

(12) Have the improvements in the areas been noted by the business?

(13) Have the improvements been backed by metrics?

\section{Literatur}

Brenner M (2006) Classifying ITIL processes. In: Proceedings of the first IEEE/IFIP international workshop on business-driven IT management (BDIM 2006)

Cater-Steel A, Tan WG, Toleman M (2006a) Challenge of adopting multiple process improvement frameworks. In: Proceedings of 14th European conference on information systems (ECIS 2006)

Cater-Steel A, Toleman M, Tan WG (2006b) Transforming IT service management - the ITIL impact. In: 17th Australasian conference on information systems, Adelaide

Cater-Steel A, Tan WG, Toleman M (2007) itSMF Australia 2006 conference: summary of ITIL adoption survey responses

Cater-Steel A, Tan WG, Toleman M (2008) itSMF Australia 2007 conference: summary of ITSM standards and frameworks survey responses

Cervone F (2008) ITIL: a framework for managing digital library services. OCLC Systems \& Services 24:87-90

Conger S, Winniford MA, Erickson-Harris L (2008) Service management in operations. In: Proceedings of the 14th Americas conference on information systems, Canada

Drucker PF, Garvin D, Leonard D, Straus S, Brown JS (1998) Harvard business review on knowledge management. Harvard Business School Press

Galup SD, Dattero R, Quan JJ, Conger S (2009) An overview of IT service management Communications of the ACM 52:124-127

Gammelgård $M$, Simonsson $M$, Lindström Å (2007) An IT management assessment framework: evaluating enterprise architecture scenarios. Information Systems and EBusiness Management 5:415-435

Hendriks L, Carr M (2002) ITIL: best practice in IT service management. The Guide to IT Service Management 1:131-150

Hochstein A, Tamm G, Brenner W (2005) Service-oriented IT management: benefit, cost and success factors. In: Proceedings of the 13th European conference on information systems, Regensburg

Huber GP (1991) Organizational learning: the contributing processes and the literatures. Organization Science 2:88-115

Iden J, Langeland L (2010) Setting the stage for a successful ITIL-adoption: a delphi study of IT-experts in the Norwegian armed forces. Information Systems Management 27:103

IT Governance Institute (2008) IT governance global status report 2008. http:// www.isaca.org/AMTemplate.cfm?Section=
ITGI_Research_Publications\&Template=/ ContentManagement/ContentDisplay. cfm\&ContentID=39735. Abruf am 200807-30

Marrone M, Kießling M, Kolbe LM (2010) Are we really innovating? An exploratory study on innovation management and service management. In: IEEE international conference on management of innovation and technology (ICMIT), 2010, pp 378-383

Orlov LM (2005) Make IT matter for business innovation. Forrester

Pollard C, Cater-Steel A (2009a) Justifications, strategies, and critical success factors in successful ITIL implementations in US and Australian companies: an exploratory study. Information Systems Management 26:164-175

Pollard C, Cater-Steel A (2009b) Justifications, strategies, and critical success factors in successful ITIL implementations in U.S. and Australian companies: an exploratory study. Information Systems Management 26:164-175

Potgieter BC, Botha JH, Lew C (2005) Evidence that use of the ITIL framework is effective. In: 18th annual conference of the national advisory committee on computing qualifications, Tauranga

Rockart JF (1982) The changing role of the information systems executive: a critical success factors perspective. Sloan Management Review 24:3-13

Rosenthal DR (1991) Meta-analytic procedures for social research revised. Sage Publications, Thousand Oaks

Spremic M, Zmirak Z, Kraljevic K (2008) IT and business process performance management: case study of ITIL implementation in finance service industry. In: Information technology interfaces, 2008 (ITI 2008), 30th international conference

Tan WG, Cater-Steel A, Toleman M, Seaniger R (2007) Implementing centralised IT service management: drawing lessons from the public sector. In: ACIS 2007 proceedings of the 18th Australasian conference on information systems, S 1060-1068

Tan W, Cater -Stel A, Toleman M (2009) Implementing IT service management: a case study focusing on critical success factors. Journal of Computer Information Systems 50:1-12

Tuttle B, Vandervelde SD (2007) An empirical examination of CobiT as an internal control framework for information technology. International Journal of Accounting. Information Systems 8:240-263

van Bon J, Jong $A D$, Kolthof $A$, Pieper $M$, Tjassing R, Veen AVD (2007) Foundations of IT service management based on ITIL V3: an introduction. Van Haren

Winniford MA, Conger S, Erickson-Harris L (2009) Confusion in the ranks: IT service management practice and terminology. Information Systems Management 26:153163

Wright TP (1936) Factors affecting the cost of airplanes. Journal of Aeronautical. Sciences 3:122-128

Young CM (2004) An introduction to IT service management. Gartner

Zarnekow R, Hochstein A, Brenner W (2005) Service-orientiertes IT-management: ITILBest-Practices und Fallstudien. Springer, Heidelberg 